\title{
Detection of human papilloma viruses in paraffin wax sections with biotinylated synthetic oligonucleotide probes and immunogold staining
}

\author{
HEATHER A CUBIE, MARY NORVAL Department of Bacteriology, University of Edinburgh \\ Medical School, Scotland
}

SUMMARY Human papilloma virus was detected by in situ hybridisation in routinely processed paraffin wax sections using a synthetically produced oligonucleotide probe, end-labelled with biotin, and amplified with anti-biotin-immunogold silver staining (anti-biotin-IGSS). This system proved more sensitive than amplification with streptavidin-biotinylated alkaline phosphatase for detecting human papilloma virus type 16 in cervical tissues. The method was successfully combined with antigen staining for papilloma virus common antigens in skin and genital warts.

This simple and quick method, using non-radioactively labelled synthetic probes, may be useful for the detection of other viruses in stored material and may be suitable for other double staining procedures.

Current methods of in situ hybridisation (ISH) for the detection of viruses rely on probes prepared from cloned nucleic acid, and often make use of expensive radiolabelled probes with limited half-lives and lengthy assay systems which are not readily adaptable to a diagnostic situation. While biotinylated probes are becoming more widely used, ${ }^{1-4}$ alternatives to cloned probes are less well documented. Recently, a method of ISH was described in which human papilloma viruses (HPV) of different types could be detected using specific synthetic oligonucleotides, produced from the known sequence data, as probes. ${ }^{5}$ We report here a modification of that technique, which was more sensitive in the detection of low copy numbers of HPV, as may be the case in cervical lesions. It was also found to be suitable for detection of viral antigen as well as viral DNA using double staining reactions.

\section{Material and methods}

Standard sections of formalin fixed, paraffin waxembedded tissues were mounted on chemically silanated slides ${ }^{6}$ and prepared for hybridisation by pretreatment with $0.2 \mathrm{M} \mathrm{HCl}$ and $0.2 \mathrm{mg} / \mathrm{ml}$ proteinase $\mathrm{K}$ (Sigma) as previously described. ${ }^{s}$ Oligonucleotide probes of 30 nucleotides in length from within the E6

Accepted for publication 16 March 1989 gene of HPV-la, HPV-6b, and HPV-16 were synthesised on an Oswel Gene Synthesiser and biotinylated using $3^{\prime}$-terminal transferase. ${ }^{5}$

Hybridisation was carried out for two hours at $42^{\circ} \mathrm{C}=$ in high stringency buffer ${ }^{4}$ and the sections washed thereafter in $2 \times \operatorname{SSC}(300 \mathrm{mM}$ sodium chloride, 30 $\mathrm{mM}$ sodium citrate, $\mathrm{pH} \mathrm{7.0)}$ three times for five minutes each. Hybridised DNA was initially detected using streptavidin-biotinylated alkaline phosphatase with nitro-blue tetrazolium and bromochloroindolyl phosphate as substrate (BRL DNA detection system, Gibco Ltd; Method 1) according to the manufacturer's instructions, except that streptavidin and conjugate incubations were increased to 20 minutes. Sections were examined without counterstaining after mounting in glycerol-gelatin. An alternative detection system was also tried (Method 2). Mouse monoclonal anti-biotin (Dako Ltd; M743) (1/20 in $5 \mathrm{mM}$ Tris$\mathrm{HCl}, \mathrm{pH} 7 \cdot 4,150 \mathrm{mM}$ sodium chloride (TBS) $+1 \%$ bovine serum albumin (BSA) (TBSB)) was applied for 45 minutes at room temperature. After two five minute washes in TBS, non-specific reactions were blocked with $20 \%$ normal rabbit serum in TBS for 10 minutes. Twenty five microlitres of rabbit anti-mouse immunoglobulin coated with colloidal gold (Dako Ltd, G385) were added at a dilution of $1 / 50$ in TBSB for one and a half hours at room temperature. The slides were washed in distilled water for 10 minutes and the deposited gold particles were enlarged using the intense II silver enhancement kit (Janssen, KN 
Biomedicals Ltd, High Wycombe), based on the immunogold silver staining method (IGSS) originally described by Holgate et al. ${ }^{7}$ Silver precipitation was observed under the microscope within 10 minutes and the reaction stopped by immersion in distilled water before "self nucleation" took over to cause high background staining.

In situ hybridisation using cloned HPV-1a, 11, and 16 DNA was carried out using detection Method $1 .^{5}$

For double staining, papilloma virus common antigens were detected before hybridisation. Sections were dewaxed and rehydrated, and endogenous peroxidase was blocked with $3 \% \mathrm{H}_{2} \mathrm{O}_{2}$ in methanol for 15 minutes. After treatment with $0.1 \%$ trypsin containing $0.1 \% \mathrm{CaCl}_{2}$ for 15 minutes at $37^{\circ} \mathrm{C}$, non-specificbinding of secondary antibody was blocked with TBS $+20 \%$ normal pig serum for 10 minutes. Rabbit antiserum to bovine papilloma virus type 1 (BPV-1) disrupted with sodium dodecyl sulphate (Dako Ltd; B580) was applied as the primary antibody (1/20 dilution in TBS $+2 \%$ normal pig serum for one hour at $37^{\circ} \mathrm{C}$ or overnight at $4^{\circ} \mathrm{C}$ ). Bound antibody was detected by sequential application of swine anti-rabbit immunoglobulin (Dako Ltd; Z196; 1/30 for 30 minutes at room temperature), anti-rabbit peroxidaseanti-peroxidase (Dako Ltd; Z113; 1/100 for $30 \mathrm{~min}$ utes at room temperature), and diaminobenzidine substrate (BDH Ltd; $0.2 \mathrm{mg} / \mathrm{ml}$ in $50 \mathrm{mM}$ Tris- $\mathrm{HCl}$, $\mathrm{pH} 7 \cdot 5$, to which was added $6 \% \mathrm{H}_{2} \mathrm{O}_{2}$ immediately before use). The reaction was stopped after five-10 minutes by washing with tap water.
Results

The feasibility of detecting HPV DNA by in situ hybridisation with biotinylated synthetic oligonucleotides was established using skin wart tissue from a hyperkeratotic hand wart and a simple hand wart. Both were strongly positive with HPV-1E6 oligonucleotide probes using Method 1 for the detection of bound probe. ${ }^{5}$ Detection Method 2 was found to be equally successful (table). Positive reactions were also obtained on tissues of vulvar and anal warts with HPV-6bE6 oligonucleotide probe using either detection method (table). These results reflected the DNA type observed with cloned probes, although the vulvar wart seemed to give more intense staining with the HPV-11 cloned probe and the anal wart with the HPV$6 \mathrm{bE6}$ probe. Double staining to detect papilloma antigens and viral DNA was tried and it proved possible to distinguish the brown colour of antigen containing cells from the grey colour of DNA containing cells (fig 1). Antigen and viral DNA were found in all four tissues.

Of seven cervical tissues exhibiting varying degrees of dysplasia, HPV antigens were not detected in any. One (specimen 8, table), which had been positive with both HPV-16 cloned probe and HPV-16E6 oligoprobe in the previous study, ${ }^{5}$ was included. It gave positive reactions when tested with HPV-16E6 oligoprobe using either detection method. A further three, however, were negative with HPV-16E6 and detection Method 1, but gave clearly positive nuclear staining

Table In situ hybridisation in four skin lesions and seven cervical lesions using HPV synthetic oligonucleotide and cloned probes

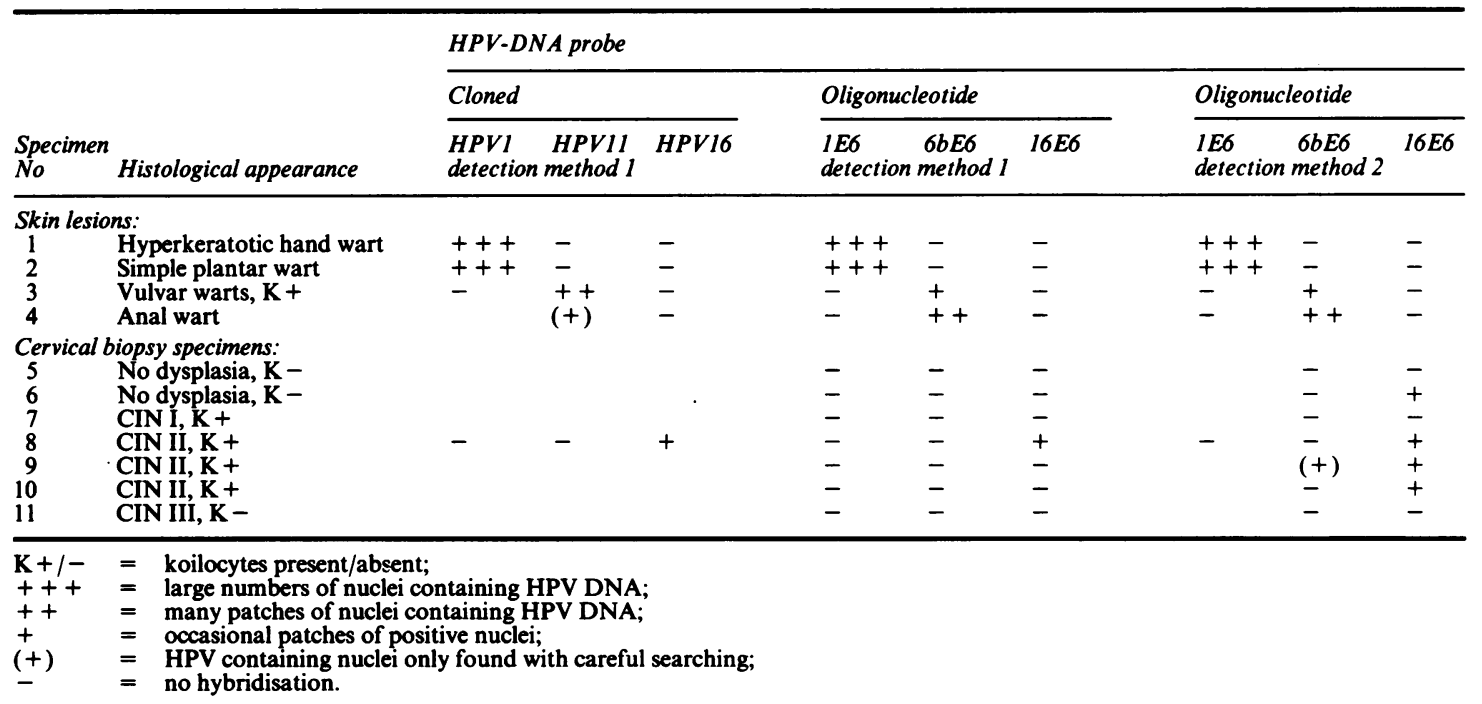




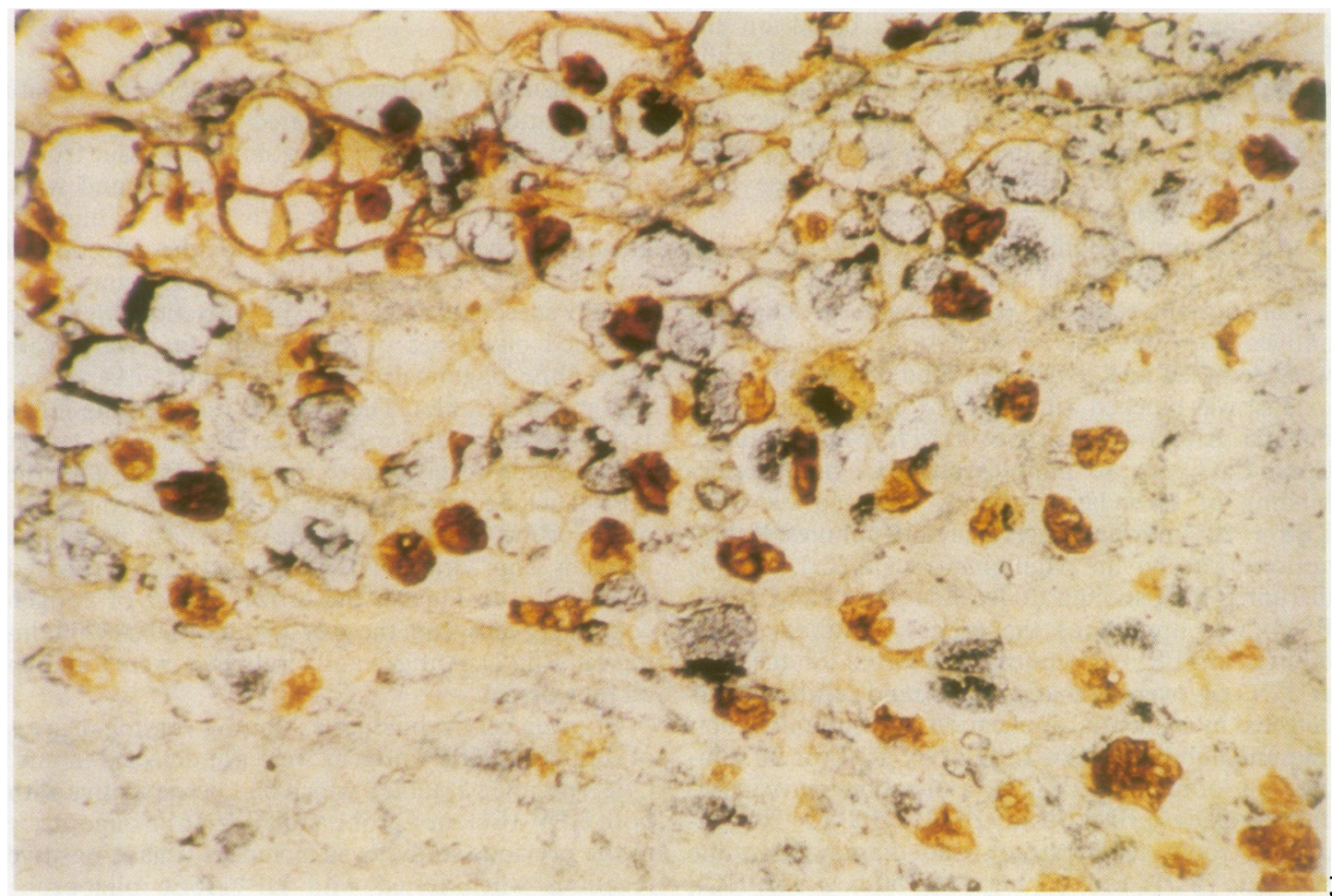

Fig 1 Section of hyperkeratotic hand wart double stained for papilloma common antigen and papilloma DNA using HPV-1E6o응 synthetic oligoprobe and anti-biotin IGSS detection. Antigen-brown; HPV-1 DNA-grey.

when detection Method 2 was used (table). Three of the four positive results were from patients with lesions of moderate dysplasia all showing koilocystosis, while the fourth (specimen 6) had shown colposcopic abnormalities that were not seen on histopathological examination. The positive reaction detectable in specimen 6 is shown in fig 2 . None of the other cervical tissues examined stained with the HPV-6bE6 oligoprobe.

\section{Discussion}

Although Southern and dot blotting are often used ${ }^{89}$ rather than in situ hybridisation for HPV detection, the combination of morphological assessment with hybridisation on routinely processed sections has particular advantages in the diagnosis of HPV infection and its association with cervical intraepithelial neoplasia. Attempts to improve the sensitivity of bound biotinylated HPV probes by amplification with additional biotin-avidin layers ${ }^{2}$ or by silver enhancement of horseradish peroxidase staining ${ }^{10}$ have been reported. Not only is the definition and localisation of DNA sequences better when obtained cytochemically, but the time required for processing and the costs are also reduced. Few diagnostic virology or histology laboratories have the facilities to make probes from cloned nucleic acid, and the introduction of synthetically produced, defined probes, either species or type specific, depending on the sequence chosen, and available in large quantities at reasonable cost, provides an attractive alternative.

In the detection of hepatitis B virus DNA in serum, Lin et al reported using a radiolabelled oligonucleotide and found equal sensitivity in a shorter time than with larger nick-translated cloned probes. ${ }^{11}$ Recently different strains of plant viroids were detected with specific radiolabelled oligoprobes, despite only a single nucleotide difference in their sequences. ${ }^{12}$ Thus radiolabelled oligoprobes seem to offer at least equal sensitivity, greater specificity, and more rapid processing than conventional probes. While a comparison of radiolabelled and biotinylated oligonucleotides has not been made, differences in sensitivity can still be obtained with different detection systems, as shown in the current study.

The anti-biotin-IGSS detection system detected HPV-16 in four of seven cervical dysplasias; the 


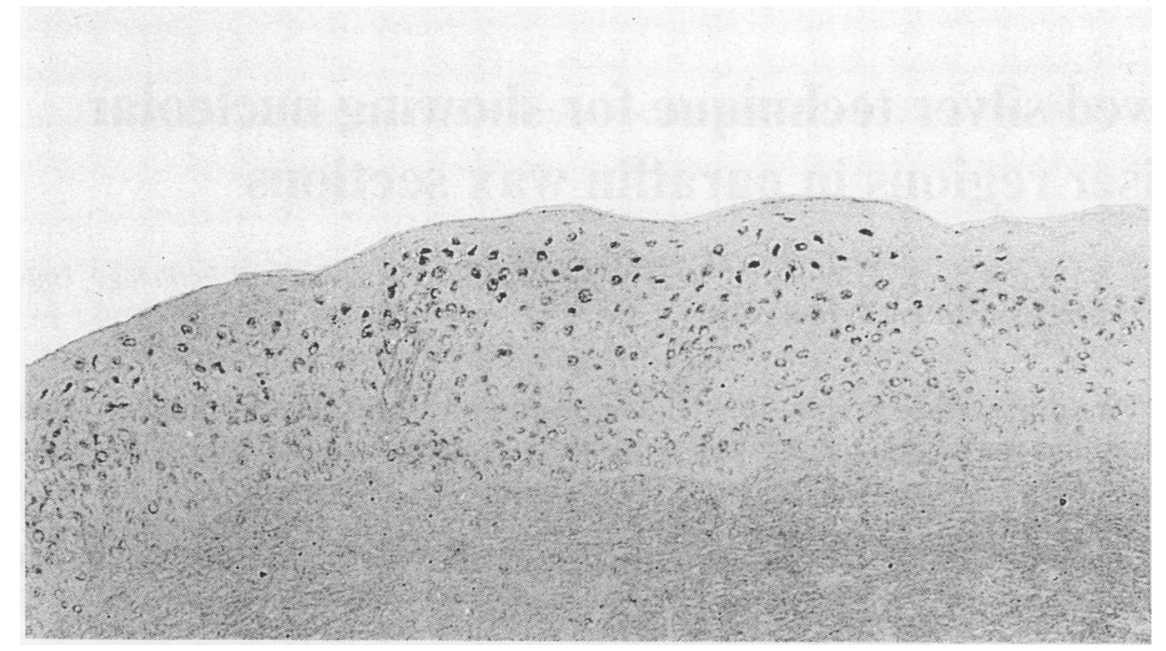

Fig 2 Section of cervical lesion showing hybridisation with HPV-16E6 synthetic oligoprobe and anti-biotin IGSS detection in specimen 6.

streptavidin-biotinylated enzyme system detected only one. Anti-biotin-IGSS has the added advantage of requiring less time for detection, particularly in the final development stages. Furthermore, by removing the streptavidin-amplification step, non-specific reactions due to endogenous biotin may be reduced. As it is now possible to biotinylate oligonucleotides at the time of synthesis, more consistent labelling should be achieved than with terminal transferase, and this may improve the sensitivity still further. While the role of $\mathrm{HPV}$ in cervical carcinogenesis is still in question, ${ }^{13}$ it is important to use the most sensitive and practical assay available to assess the biological importance of its presence in normal, dysplastic, and malignant lesions.

We thank Drs GE Smart, S Fletcher, and the staff of the Lothian Area Colposcopy Clinic for providing access to their patients; Mr K Rae and his staff in the department of pathology for cutting sections; and Dr T Brown and Ms S Thomson, department of chemistry, University of Edinburgh, for producing the synthetic oligonucleotides. We thank Dako Ltd for providing the anti-biotin and rabbit anti-mouse immunogold.

The financial support of the Cancer Research Campaign is gratefully acknowledged.

\section{References}

1 Brigati DJ, Myerson D, Leary JJ, et al. Detection of viral genomes in cultured cells and paraffin embedded tissue sections using biotin-labelled hybridisation probes. Virology 1983;126:32-50.

2 Beckmann AM, Myerson D, Daling JR, Kiviat BN, Fenoglio CM, McDougall JK. Detection and localisation of human papillomavirus DNA in human genital condylomas by in situ hybridisation with biotinylated probes. J Med Virol 1985; 16:265-73.

3 Forghani B, Dupuis KW, Schmidt NJ. Rapid detection of herpes simplex virus DNA in human brain tissue by in situ hybridisation. J Clin Microbiol 1985;22:656-8.

4 Ungar ER, Budgeon LR, Myerson D, Brigati DJ. Viral diagnosis by in situ hybridisation. Am J Surg Pathol 1986;10:1-8.

5 Cubie HA, Norval M. Synthetic oligonucleotide probes for the detection of human papilloma viruses by in situ hybridisation. $J$ Virol Methods 1988;20:239-49.

6 Tourtellotte WN, Verity AN, Schmid P, Martinez S, Shapshak P. Covalent binding of formalin fixed paraffin embedded brain tissue sections to glass slides suitable for in situ hybridisation. $J$ Virol Methods 1987;15:87-99.

7 Holgate CS, Jackson P, Cowen P, Bird CC. Immunogold silver staining - new method of immunostaining with enhanced sensitivity. J Histochem Cytochem 1983;31:938-44.

8 McCance DJ, Campion MJ, Chesters PM, Jenkins D, Singer A. Prevalence of human papillomavirus type 16 DNA sequences in cervical intraepithelial neoplasia and invasive carcinoma of the cervix. Br J Obstet Gynaecol 1985;92:1101-5.

9 Meanwell CA, Cox MF, Blackledge G, Maitland NJ. HPV-16 DNA in normal and malignant cervical epithelium: implications for the aetiology and behaviour of cervical neoplasia. Lancet 1987;i:703-7.

10 Burns J, Graham AK, Frank C, Fleming KA, Evans MF, McGee JO'D. Detection of low copy human papillomavirus DNA and mRNA in routine paraffin sections of cervix by non-isotopic in situ hybridisation. J Clin Pathol 1987;40:858-64.

11 Lin HJ, Wu P-C, Lai C-L. An oligonucleotide probe for the detection of hepatitis B DNA in serum. J Virol Methods 1987;15:139-49.

12 Sano T, Kudo H, Sugimoto T, Shikata E. Synthetic oligonucleotide hybridisation probes to diagnose hop stunt viroid strains and citrus exocortis viroid. J Virol Methods 1988;19: 109-20.

13 Kitchener HC. Commentary: Does HPV cause cervical cancer? Br J Obstet Gynaecol 1988;95:1089-91.

Requests for reprints to: Dr Heather A Cubie, Department of Bacteriology, University of Edinburgh Medical School, Teviot Place, Edinburgh EH8 9AG, Scotland. 